\title{
Feasibility and first results of a group program to increase the frequency of cognitively stimulating leisure activities in people with mild cognitive impairment (AKTIVA-MCl)
}

\author{
This article was published in the following Dove Press journal: \\ Clinical Interventions in Aging \\ 12 September 2017 \\ Number of times this article has been viewed
}

\author{
Valentina A Tesky' \\ Theresa Köbe ${ }^{2}$ \\ A Veronica Witte 2,3 \\ Agnes Flöel ${ }^{2}$ \\ Jan Philipp Schuchardt ${ }^{4}$ \\ Andreas Hahn ${ }^{4}$ \\ Johannes Pantel' \\ 'Geriatric Medicine, Institute of \\ General Practice, Goethe University, \\ Frankfurt, Germany; ${ }^{2}$ Department \\ of Neurology, Charité - University \\ Medicine Berlin, Berlin, Germany; \\ ${ }^{3}$ Department of Neurology, \\ Max Planck Institute of Human \\ Cognitive and Brain Sciences, \\ University of Leipzig, Leipzig, \\ Germany; ${ }^{4}$ Department of Nutrition \\ Physiology and Human Nutrition, \\ Gottfried Wilhelm Leibniz University, \\ Hannover, Germany
}

\begin{abstract}
AKTIVA-MCI is a program for patients with mild cognitive impairment (MCI) that aims to enhance participation in cognitively stimulating leisure activities. Participation in cognitively stimulating activities seems to be a potential strategy for people with MCI delaying cognitive decline for a while. In total, $35 \mathrm{MCI}$ patients were enrolled in the pilot study of whom 29 completed the whole program (16 female, 71.1 \pm 7.5 years; Mini Mental Status Examination score: $28 \pm 2.2$ ). Daily activity protocols were used to measure the frequency of participation in cognitively stimulating activities during the program (12 sessions). Additional standardized psychometric tests and questionnaires were used to assess cognition, mood, and subjective memory decline. Analyses of the daily activity protocols showed that during the intervention participants increased the frequency of several cognitively stimulating leisure activities. Comparison of pre-post data indicates no changes in cognitive status, mood, and subjective memory decline. These findings indicate that the program is suitable for patients with MCI.

Keywords: older people, MCI, pilot study, intervention study, cognitively stimulating leisure activities, training program, daily activity protocols
\end{abstract}

\section{Introduction}

Demographic change is expected to increase the number of older individuals with mild cognitive impairment (MCI). MCI is a risk syndrome, and in some individuals, it describes the clinical transition between normal cognitive aging and early dementia. ${ }^{1}$ Currently, there are no established pharmacological treatment approaches for MCI. Strategies to treat MCI are the same as those aimed at preventing cognitive decline in cognitively normal older people and include the treatment of lifestyle-related chronic diseases (eg, hypertension and hypercholesterolemia), physical exercise, a Mediterranean diet, and cognitive activity. ${ }^{2}$ To enhance cognitive activity and to improve cognitive function, many cognitive interventions have been conducted on people suffering from objective memory decline. These trainings usually consist of guided exercises involving specific cognitive tasks and memory techniques. ${ }^{3-5}$ There is some evidence that cognitive training can be effective in improving various aspects of cognitive function such as memory, attention, and psychological well-being. ${ }^{6}$ But most of the training programs are limited in terms of transferability and sustainability. ${ }^{4,5,7,8}$ Martin et al and Reijnders et al concluded that there is currently little evidence that memory interventions help people with MCI. ${ }^{4,5}$ For this reason, a different approach to enhancing 
cognitive activity in individuals with MCI is attracting interest. Interventions are required that take account of how people function in an everyday context. ${ }^{9}$ Findings from the studies of Scarmeas et al and Wilson et al have indicated that frequent participation in cognitively stimulating leisure activities like reading, playing chess, or making music in middle age can reduce the risk of cognitive decline in the future. ${ }^{11,12}$ There is a correlation between participation in these activities and a reduced risk of incident dementia. Participation in these cognitively stimulating activities as well as participation in productive activities like gardening, cooking, and knitting also seems to be a potentially effective strategy for the prevention of cognitive decline in people with MCI. But for patients with MCI, it is important that interventions involving cognitive training programs include interactive elements. ${ }^{9}$ These could take the form of practical problemsolving strategies, self-assertiveness training or stress management. Selective memory training does not improve everyday performance in people with MCI. ${ }^{10}$ For this reason, integrative concepts - referred to by the term cognitive rehabilitation - are preferable. These approaches combine different therapeutic measures with the aim of improving daily competence and minimizing the consequences of cognitive disorders. Kasper et al postulate that the efficacy of cognitive rehabilitation in MCI and mild Alzheimer's disease (AD) is mainly dependent on four factors, namely individuality (consideration of personal needs), compensation (strategies to compensate for cognitive impairment), interaction (inclusion of relatives and environmental conditions), and integration (integration of various therapeutic methods). ${ }^{10}$ Also, cognitive rehabilitation approaches should provide compensatory possibilities for clearly defined routine challenges and the individual needs of those affected.

The approach of the AKTIVA program (active cognitive stimulation - prevention for elderly people: German: Aktive Kognitive Stimulation - Vorbeugung im Alter) which is presented in this study focuses on the study results from Scarmeas et al and Wilson et al. ${ }^{11,12}$ The AKTIVA training program is based on the concept of cognitive activation through cognitively stimulating leisure activities. This approach is an innovative one and has not been used previously in intervention studies for people with MCI. Although Dannhauser et al designed a complex activity intervention for people with MCI by using physical activity, group-based cognitive stimulation, and individual cognitive stimulation, AKTIVA-MCI is different from this intervention. ${ }^{13}$ In contrast to this complex activity intervention, the AKTIVAMCI program has no exercise of arts and crafts activities all together and no training of specific cognitive functions.
Furthermore, AKTIVA-MCI takes into account the four aforementioned factors that Kasper et al describe as factors that determine efficacy (individuality, compensation, interaction, and integration). ${ }^{10}$

AKTIVA was first established and evaluated in healthy older adults and has now been modified for patients with MCI. ${ }^{14-16}$ The AKTIVA-MCI program is designed for small groups and is resource oriented. In the present study, we report results of a pilot study using AKTIVA-MCI as an intervention in a group of 29 patients with multi-domain amnestic MCI. The following main hypotheses were investigated:

- The first hypothesis was that the program is feasible. We assumed that participants were motivated to take part in such a training program, and they were interested in implementing the content of the program in their daily routines.

- The second hypothesis was that participants in the intervention training program would increase their participation in leisure activities involving cognitive stimulation.

- The third hypothesis was that the outcome measures of cognitive function, mood, and attitude toward aging would improve or remain stable during the participation in the program.

\section{Methods}

\section{Participants}

We confirm that this study was approved by the local ethics committee of University Hospital Charité, Berlin, and that all the participants provided informed consent. In total, 35 participants (19 female; $71.6 \pm 7$ years; Mini Mental Status

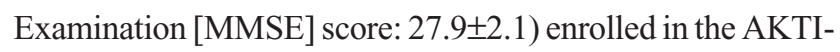
VA-MCI program. The participants had amnestic MCI and were recruited in Berlin and Frankfurt am Main, Germany. The settings for the recruitment were the memory clinic of the Department of Neurology of the Charité University Hospital and Neurology specialist practice in Berlin and the Geriatric Medicine, Institute of General Practice of Goethe University in Frankfurt am Main. Inclusion criteria were diagnosis of amnestic MCI within 12 months before baseline visits, diagnosed according to Mayo criteria based on a subjective cognitive complaint, and an objective memory impairment in a standardized test (performing at least $1 \mathrm{SD}$ below age- and education-specific norm in relevant subtest of the CERAD-Plus test or the AVLT test battery [Total Word List, Delayed Recall Word List/Figures]), no impairment in activities of daily living, and no dementia. ${ }^{17,18}$ On average, $80 \%$ of patients had a deficit in the verbal domain and $50 \%$ in the visuoconstructive domain. Exclusion criteria comprised MMSE score $\leq 24$ at baseline visits and history of severe 
untreated medical, neurological, and psychiatric diseases, type 2 diabetes mellitus, stroke or brain pathologies identified in a MRI scan, specifically, stroke and tumors, intake of cholinesterase inhibitors or memantine, and not fluent in German. Psychiatric comorbidity was monitored by using Beck's Depression Inventory and the State-Trait Anxiety Inventory (STAI X1). ${ }^{19,20}$

Of the 35 participants with amnestic MCI, only 29 completed the whole intervention, including post measure-

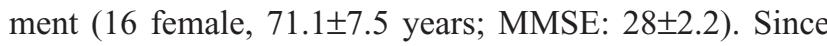
the AKTIVA-MCI participants were a subsample of a larger study on the effects of a combined intervention in patients with MCI, some of them enjoyed an additional sport intervention which included regular aerobic exercise (stationary biking sessions of 30-45 min, twice a week). Accordingly, participants were randomly divided into two groups of approximately equal size. Fourteen participants in intervention group 1 took part in only the AKTIVA-MCI

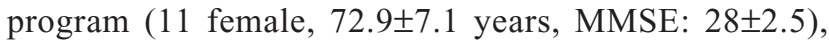
whereas the 15 participants of intervention group 2 ( 5 female,

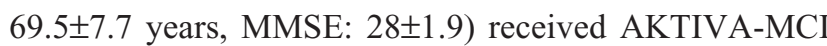
and additional aerobic exercise. Moreover, participants of both the intervention groups took dietary supplements with omega-3 fatty acids.

\section{Design and study description}

This intervention study without a control group considers only the AKTIVA-MCI subgroup of the Bundesministerium für Bildung und Forschung project "Enhancing memory functions in patients with mild cognitive impairment by dietary interventions and in combination with exercise and cognitive training - Proof of concept and mechanisms" (FKZ 01EA1328). For this reason, only participants in the AKTIVAMCI program $(\mathrm{N}=35)$, including the assessment and the main results of the intervention, were described. Köbe et al provide further information on the whole research project. ${ }^{21}$

Table 1 presents the flowchart for the AKTIVA-MCI substudy. After neuropsychological baseline assessment (pretest), all the participants received 12 training sessions. After the final intervention session, neuropsychological test was performed again in all the participants (posttest). Participants did not receive weekly intervention treatment from the 11th to the 13th week because of individual coaching. As part of the AKTIVA-MCI program, every participant was offered individual coaching for $\sim 45$ minutes. The coaching was led by a professional psychologist trained for the AKTIVA-MCI program. In these meetings, the participants spoke about their individual participation in more cognitively stimulating activities in their leisure time. The execution of these activities was planned in detail, and systematic approaches to monitor their success were discussed. The coaching was scheduled for 2-3 weeks to ensure that all the participants had the chance to take part. The intervention then continued with four regular group sessions over the next 4 weeks. This was followed by another 4-week period from the 18 th to the 22 nd week, during which neither group sessions nor individual coaching took place. This so-called training phase should give all participants the opportunity to implement strategies and information from the training sessions into their daily lives. The intervention concluded with one booster session during week 23 . In this session, all experiences and/or problems from the training phase were discussed with the participants. All the participants received post testing during the 24th and the final week of the study.

As stated earlier, participants in the intervention group 2 had additional sport training sessions for 45 minutes, twice a week. These were supervised by professional exercise instructors. They always started training with a 4-minute warm-up ( $40 \%$ intensity of anaerobic threshold), then the intensity was gradually increased until patients were exercising at an intensity of $80 \%$, followed by a cooldown period of 6 minutes (40\% intensity). Training at an intensity of $80 \%$ was maintained throughout the study.

Köbe et al provide further information on the sport training sessions. ${ }^{21}$

\section{Assessment of feasibility}

After the AKTIVA-MCI training program, all the participants were asked to evaluate the program in terms of feasibility.

Table I Flowchart of the AKTIVA-MCI intervention program

\begin{tabular}{|c|c|c|c|c|c|c|c|}
\hline \multirow[t]{2}{*}{ Group } & \multicolumn{7}{|l|}{ Weeks } \\
\hline & $1-3$ & $4-10$ & $11-13$ & |4-17 & $18-22$ & 23 & 24 \\
\hline $\begin{array}{l}\text { IG_I } \\
(\mathrm{N}=|4, \mathrm{I}| \text { female })\end{array}$ & Pretest & Sessions I-7 & Individual coaching & Sessions 8-11 & Break & Booster session & Posttest \\
\hline \multirow[t]{2}{*}{$\begin{array}{l}\text { IG_2 } \\
(\mathrm{N}=15,5 \text { female })\end{array}$} & Pretest & Sessions I-7 & Individual coaching & Sessions 8-II & Break & Booster session & Posttest \\
\hline & \multicolumn{7}{|c|}{ Additional stationary biking sessions of $30-45 \mathrm{~min}, 2$ times a week } \\
\hline
\end{tabular}

Note: IG_I, intervention group I (AKTIVA-MCI); IG_2, intervention group 2 (AKTIVA-MCI + sport).

Abbreviation: AKTIVA-MCl, active cognitive stimulation - prevention for elderly people - mild cognitive impairment. 
For this purpose, the questionnaire that is used in regular AKTIVA training with healthy older people was used. ${ }^{14}$ Satisfaction with the program was measured based on 13 items. Participants were asked to evaluate the content and quality of information, the atmosphere in the group, and about their overall impression of the program. Furthermore, they were asked whether they would recommend AKTIVA-MCI and what they regarded as the most important (learning) experience gained by participants during the group sessions.

To measure the frequency of participation in cognitively stimulating activities during the intervention period, participants were required to complete daily activity protocols. These protocols were 30-item questionnaires that measured the frequency of participation in leisure activities on a 6-point Likert scale. This questionnaire was created on the basis of items reported in the retrospective studies of Wilson et al, Wilson, Scherr et al, and Scarmeas et al and is an advanced version of the weekly activity protocols first used by Tesky et al. ${ }^{11,12,15,22}$ Participants were given a further battery of neuropsychological tests and questionnaires before and after the AKTIVA-MCI training program. These instruments were also used in the original AKTIVA-study. ${ }^{15}$ Cognition was measured by using the MMSE, and the cognitive part of the Alzheimer's Disease Assessment Scale (ADAS-Cog). ${ }^{23,24}$ The test battery comprised self-reported questionnaires such as the Self-Rating Depression Scale (SDS), Memory Complaint Questionnaire (MAC-Q), Nuremberg Self-Rating List (Nürnberger Selbsteinschätzungsliste, NSL), Philadelphia Geriatric Center Morale Scale (PGCMS) and quality-of-life scale (Skala zur Lebenszufriedenheit, LBZ). ${ }^{25-28}$

\section{Training description}

The AKTIVA-MCI intervention is a training program specifically developed for the needs of people with MCI. In contrast to standard cognitive interventions (eg, cognitive training), cognitive enhancement in this program takes place through the promotion of cognitively stimulating leisure activities like reading, drawing, making music, or playing chess. ${ }^{5}$ The focus of AKTIVA is on increasing the frequency of participation in these activities. The program is based on the AKTIVA program (active cognitive stimulation - prevention of cognitive decline in the elderly) for cognitively healthy elderly people but was modified to take account of the special needs of individuals with MCI. The AKTIVA-MCI program was also described in a standardized manual designed to increase cognitive stimulation in everyday life and during leisure time. The manual gives a detailed description on how to perform the group sessions and the coaching sessions and is publicly available. ${ }^{29}$

Table 2 gives an overview on the content of the AKTIVAMCI training modules. In short, the training program consists of three modules, namely "Psychoeducation," "Activation \& Coaching," and "Booster," and covers all together 12 sessions of 120 minutes. In small groups of 6-10 individuals, participants are informed about the syndrome MCI, cognitive decline in the elderly, risk factors, and individual prevention strategies for cognitive decline. Participants are also taught about other topics related to aging, that is, loss of sensory abilities, experience of life, and free-time in retirement. Participants are provided with information on the importance and principles of motivation, self-motivation, and self-awareness. Games and different exercises such as role play, coaching cards, memory tasks, or a work-of-art game enrich the theoretical modules provided in the group sessions. In addition, there is always time for discussion. Participants are further instructed about coping strategies to combat impairments and restrictions in daily life, as well as strategies to improve quality of life and prevent social isolation. A core element of the program is that participants are systematically instructed and motivated to perform cognitively stimulating activities like reading, playing games, and making music, as part of their daily routines. They are told about the positive effects of these cognitive leisure activities, as well as the association that

Table 2 Content of the AKTIVA-MCI training modules

\begin{tabular}{|c|c|c|c|c|}
\hline \multicolumn{5}{|l|}{ Weeks } \\
\hline $1-7$ & $8-10$ & $1|-| 4$ & $15-18$ & 19 \\
\hline $\begin{array}{l}\text { Psychoeducation } \\
\text { sessions I-7 }\end{array}$ & Individual coaching & $\begin{array}{l}\text { Activation and coaching } \\
\text { sessions } 8 \text { - I I }\end{array}$ & Break & $\begin{array}{l}\text { Booster } \\
\text { session } 12\end{array}$ \\
\hline Topics & Individual coaching & Topics & No intervention & Topics \\
\hline - Memory models & & - Motivation & treatment & - Exchange of experience \\
\hline - Risk factors for $\mathrm{MCl}$ & & - Coping strategies & & - Discussion of success \\
\hline - Prevention of $\mathrm{MCl}$ & & - Attribution styles & & and problems \\
\hline - Individual health status & & - Memory management & & - News about $\mathrm{MCl}$ \\
\hline - Goal setting & & & & - Future goals \\
\hline
\end{tabular}

Abbreviations: $\mathrm{MCl}$, mild cognitive impairment; AKTIVA-MCI, active cognitive stimulation - prevention for elderly people - mild cognitive impairment. 
seems to exist between an active lifestyle and cognitive decline. Furthermore, emphasis was consistently placed on social contacts.

The translation of the newly acquired knowledge and skills into daily routine is supported by individual coaching. By analyzing the lifestyle in individual sessions, the capabilities and capacities of participants to enhance cognitively stimulating activities are discussed. Furthermore, individual motivational strategies to participate in these activities are introduced. There were no rigid guidelines prescribing specific activities. Participants are expected to voluntarily change their behavior and independently increase the frequency of their participation in leisure activities involving cognitive stimulation. Aim of the program is to cause a change of behavior in a self-directed manner. In the final session, the so-called booster session that took place after a break in the intervention of 4-5 weeks, participants were given the opportunity to discuss their progress toward previously identified goals.

\section{Outcome measures}

The primary outcomes were the feasibility of the training program and the frequency of participation in cognitively stimulating activities (Figure 1). Secondary outcomes were cognition, mood, subjective memory decline, and attitude to old age. The feasibility of AKTIVA-MCI was assessed at the

\begin{tabular}{|c|c|c|c|c|c|c|}
\hline $\begin{array}{l}\text { Please mark, how often you have participated } \\
\text { in the following activities today... }\end{array}$ & Not at all & $\begin{array}{l}\text { Less than } \\
30 \text { min }\end{array}$ & $\begin{array}{l}\text { More than } \\
30 \text { min }\end{array}$ & 1 hour & 2 hours & $\begin{array}{l}\text { More than } \\
2 \text { hours }\end{array}$ \\
\hline$\ldots$ watched TV. & & & & & & \\
\hline a) News & ( ) & ( ) & ( ) & ( ) & ( ) & ( ) \\
\hline b) Entertainment program & ( ) & ( ) & ( ) & ( ) & ( ) & ( ) \\
\hline ... read a book/books. & ( ) & ( ) & ( ) & ( ) & ( ) & ( ) \\
\hline ... read a magazine/newspaper. & & & & & & \\
\hline a) Daily, weekly newspaper & ( ) & ( ) & ( ) & ( ) & ( ) & ( ) \\
\hline b) Journal, magazine & ( ) & ( ) & ( ) & ( ) & ( ) & ( ) \\
\hline ... played a game. & & & & & & \\
\hline a) With others (chess, cards, etc) & ( ) & ( ) & ( ) & ( ) & ( ) & ( ) \\
\hline b) Alone or on the PC (eg, solitaire) & ( ) & ( ) & ( ) & ( ) & ( ) & ( ) \\
\hline $\begin{array}{l}\text {... worked at the PC (internet, writing letters, } \\
\text { etc) }\end{array}$ & ( ) & ( ) & ( ) & ( ) & ( ) & ( ) \\
\hline ... done gardening. & ( ) & ( ) & ( ) & ( ) & ( ) & ( ) \\
\hline ... played a sport. & & & & & & \\
\hline a) Club-/group sport & ( ) & ( ) & ( ) & ( ) & ( ) & ( ) \\
\hline $\begin{array}{l}\text { b) Individual sport (fitness center, jogging, } \\
\text { walking, swimming, cycling) }\end{array}$ & ( ) & ( ) & ( ) & ( ) & ( ) & ( ) \\
\hline c) Recreational sport like yoga & ( ) & ( ) & ( ) & ( ) & ( ) & ( ) \\
\hline $\begin{array}{l}\text {... participated in further training courses } \\
\text { (language course, university). }\end{array}$ & ( ) & ( ) & ( ) & ( ) & ( ) & ( ) \\
\hline ... walked. & & & & & & \\
\hline a) Done errands (shopping, bank, post office) & ( ) & ( ) & ( ) & ( ) & ( ) & ( ) \\
\hline b) Gone for a walk, taken dog for a walk & ( ) & ( ) & ( ) & ( ) & ( ) & ( ) \\
\hline
\end{tabular}

Figure I The daily activity protocol (extracts). 
end of the sessions by means of an evaluation questionnaire (Figure 2). Activity protocols had to be completed daily during the intervention period. The psychometric tests were used as pre- and post-tests.

To measure the frequency of participation in cognitively stimulating activities during the intervention training program, participants in the intervention groups were required to complete daily activity protocols. The daily activity protocols were a further development of the weekly activity protocols used in the first AKTIVA study, and a 30-item questionnaire was used. ${ }^{15}$ The theoretical basis for the selection of items was given by the items reported in the retrospective studies of Wilson et al, Wilson, Scherr et al and Scarmeas et al. ${ }^{11,12,22}$ Participants indicated the frequency of their participation in different leisure activities like reading, playing games, doing sports, making music, or meeting friends on a 6-point Likert scale (Figure 1).

The participants also completed an evaluation questionnaire (Figure 2), which consists of 13 items. For example, the participants assessed the atmosphere of the group sessions, the information provided, and the trainers/facilitators' competence. Open-ended questions were also used to record any individual benefit enjoyed by the participant.

\section{Data analysis}

Data from the evaluation questionnaire were analyzed descriptively. The data from daily activity protocols were analyzed using trend analysis, which examined whether the

\begin{tabular}{|c|c|c|c|c|}
\hline \multicolumn{5}{|l|}{ Dear participant, } \\
\hline \multicolumn{5}{|c|}{$\begin{array}{l}\text { We are interested in how helpful you found the group program, how much you enjoyed it and } \\
\text { whether you have any further suggestions. We would therefore kindly like to ask to answer } \\
\text { the following questions. }\end{array}$} \\
\hline \multirow{2}{*}{\multicolumn{5}{|c|}{ By completing this form, you will help us develop the group program and improve its quality. }} \\
\hline & & & & \\
\hline & $\begin{array}{l}\text { I completely } \\
\text { agree }\end{array}$ & $\begin{array}{l}\text { I mostly } \\
\text { agree }\end{array}$ & $\begin{array}{l}\text { I mostly } \\
\text { disagree }\end{array}$ & $\begin{array}{l}\text { I completely } \\
\text { disagree }\end{array}$ \\
\hline a) Interesting & ( ) & ( ) & ( ) & $($ ) \\
\hline b) Understandable & () & () & ( ) & ( ) \\
\hline c) Informative & ( ) & ( ) & ( ) & $($ ) \\
\hline $\begin{array}{l}\text { d) Applicable in } \\
\text { everyday life }\end{array}$ & $($ ) & ( ) & ( ) & $($ ) \\
\hline $\begin{array}{l}\text { e) Clear and well } \\
\text { structured }\end{array}$ & ( ) & ( ) & ( ) & ( ) \\
\hline \multicolumn{5}{|c|}{ f) I would have liked to receive information on: } \\
\hline \multicolumn{5}{|c|}{ 2. How would you rate the content and quality of the distributed information sheets? } \\
\hline \multicolumn{5}{|c|}{ ( ) Very good ( ) Good ( ) Should be reviewed ( ) Not good at all } \\
\hline \multicolumn{5}{|c|}{ 3. How would you rate the atmosphere in the group? } \\
\hline & $\begin{array}{l}\text { I completely } \\
\text { agree }\end{array}$ & $\begin{array}{l}\text { I mostly } \\
\text { agree }\end{array}$ & $\begin{array}{l}\text { I mostly } \\
\text { disagree }\end{array}$ & $\begin{array}{l}\text { I completely } \\
\text { disagree }\end{array}$ \\
\hline a) Pleasant & ( ) & ( ) & ( ) & ( ) \\
\hline b) Trusting & ( ) & $($ ) & ( ) & ( ) \\
\hline $\begin{array}{l}\text { c) Any uncertainties } \\
\text { were clarified where } \\
\text { necessary }\end{array}$ & ( ) & ( ) & ( ) & ( ) \\
\hline $\begin{array}{l}\text { d) Questions were } \\
\text { taken seriously and } \\
\text { answered }\end{array}$ & ( ) & ( ) & ( ) & ( ) \\
\hline \multicolumn{5}{|c|}{ 4. How would you rate the group program overall? } \\
\hline ( ) Excellent ( ) Goc & ( ) Fair ( & oor & & \\
\hline
\end{tabular}

Figure 2 The evaluation questionnaire (extract). 
Table 3 Baseline characteristics and pretest values of both the intervention groups

\begin{tabular}{|c|c|c|c|c|c|}
\hline Variable & IG_I $(\mathbf{N}=\mid 4)$ & IG_2 (N=I5) & df & $\mathbf{F} / \chi^{2}$ & $P$-value \\
\hline Age, mean (SD) & $72.9(7)$ & $69.5(7.7)$ & $1 / 27$ & 1.5 & 0.24 \\
\hline Women, no (\%) & II (78.6) & $5(33.3)$ & 1 & 5.9 & 0.01 \\
\hline Years of education, mean (SD) & II.I (I.9) & $\mathrm{II} .4(2.1)$ & 4 & 4.9 & 0.30 \\
\hline Academic education, no (\%) & $3(21.4)$ & $9(60)$ & 1 & 4.4 & 0.04 \\
\hline MMSE, mean (SD) & $28(2.5)$ & $28(1.9)$ & $\mathrm{I} / 27$ & 0.0 & 1.0 \\
\hline MMSE, range & $22-30$ & $23-30$ & & & \\
\hline ADAS-Cog, mean (SD) & $7.1(3.7)$ & $9.1(3.5)$ & $1 / 27$ & 2.2 & 0.15 \\
\hline ADAS-Cog, range & $2-18$ & $5-17$ & & & \\
\hline SDS, mean (SD) & $35.9(9.1)$ & $37.5(8.3)$ & $1 / 27$ & 0.3 & 0.62 \\
\hline NSL, mean (SD) & $45.2(10)$ & $48.9(12.7)$ & $1 / 27$ & 0.7 & 0.40 \\
\hline MAC-Q, mean (SD) & $25(4.1)$ & $27.4(4.7)$ & $1 / 27$ & 2.1 & 0.16 \\
\hline PGCMS, mean (SD) & $10.5(4.5)$ & $12.3(3.7)$ & $1 / 27$ & 1.5 & 0.24 \\
\hline LBZ, mean (SD) & $25.4(3.5)$ & $25.9(4.9)$ & $\mathrm{I} / 27$ & 0.1 & 0.75 \\
\hline
\end{tabular}

Note: IG_I, intervention group I (AKTIVA-MCI); IG_2, intervention group 2 (AKTIVA-MCI + sport).

Abbreviations: MMSE, Mini Mental Status Examination; ADAS-Cog, Alzheimer's Disease Assessment Scale cognitive subscale; SDS, Self-Rating Depression Scale; NSL, Nuremberg Self-Rating List; MAC-Q, Memory Complaint Questionnaire; PGCMS, Philadelphia Geriatric Center Morale Scale; LBZ, quality-of-life-scale; df, degree of freedom; AKTIVA-MCl, active cognitive stimulation - prevention for elderly people - mild cognitive impairment; $\mathrm{F}$, F-value; $\chi^{2}$, Chi-square-value.

observed activity variables showed a linear trend. For this purpose, the activity items were first standardized and then aggregated. The analysis included the activity protocols for 16 weeks (112 days). However, only data for participants who completed all 16 weekly activity protocols were included in the trend analysis $(\mathrm{N}=24)$.

For the presentation of the results of the trend analyses, the sample was presented as a whole because there were no significant differences between the two interventional groups with respect to baseline characteristics and pretest values (Table 3 ).

An analysis of variance with repeated measures was also used to evaluate the effects of the AKTIVA-MCI intervention. There were no significant pretest differences in cognitive performance, mood, and attitude toward aging between the two groups (Table 3). Accordingly, for the analysis of prepost differences, data from intervention groups 1 and 2 were pooled and analyzed together.

\section{Results}

A summary of the baseline characteristics and the pretest differences of both the intervention groups is provided in Table 3.

Fifty-five percent of participants were women, and the mean age was 71.1 years $( \pm 7.5$ years). Half of the participants had attended school for $\sim 13$ years $(51.7 \%)$ and nearly half of the participants $(41.4 \%)$ had completed a university education. Pretest scores measured using the cognitive tests (MMSE and ADAS-Cog) were mostly in a range indicating pathological cognitive decline, although a few participants had test scores in the normal range of 28-30 on the MMSE and 0-9 on the ADAS-Cog. Pretest scores measured using the SDS showed no indication that a patient was suffering from depression. Pretest scores measured using the NSL indicated that participants in the study showed age-related decline pertaining to vitality, cognition, and social interaction. The participants' pretest scores on the MAC-Q, which measures subjective memory decline, indicated that participants in the study showed slight to medium age-related decline in memory function. Participants' pretest scores on the PGCMS showed that the attitude of participants toward the elderly is positive rather than negative. Participants' pretest scores on the LBZ showed that participants had a high quality of life. There were two significant sample differences between both the groups; the intervention group 1 consisted of more women while more participants in the intervention group 2 group had an academic education. Because there were no group differences for the test scores, these findings were considered to be of no relevance.

Overall, 29 participants completed the evaluation questionnaire. All the participants were pleased with the training and $94 \%$ would recommend it to others. The majority rated the provided information as interesting and understandable. Figure 3 shows the exact percentages for these items. Overall, 84\% described the atmosphere as positive, and 91\% said the group instructors had actively involved participants in the training and addressed them personally. Individual benefits of the training included greater interest in participating in more activities on a daily basis, personal support and a feeling one is not alone with cognitive decline, no longer feeling ashamed because of forgetfulness, and feeling motivated and reassured.

The analyses of the weekly activity protocols of the participants ( $\mathrm{N}=24$; Table 4) showed significant outcomes during the 112 days of the intervention program (weeks 1-16). All the participants showed significant reduction in the frequency 


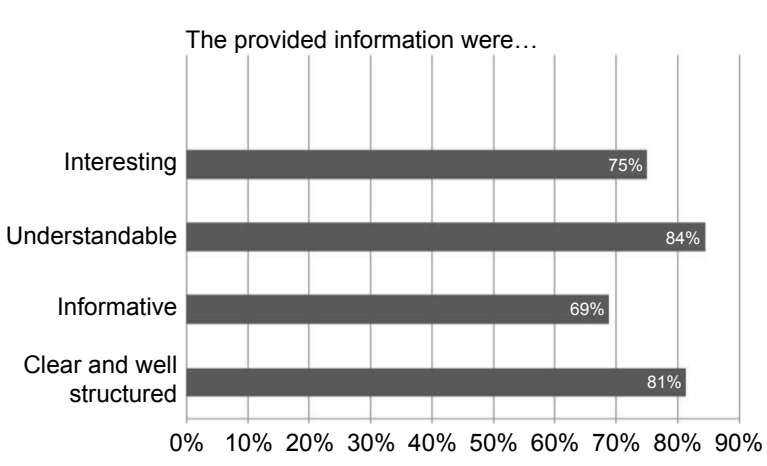

Figure 3 Ratings about the provided information.

of participation in some leisure activities that have little cognitive benefit (eg, watching television; Figure 4) and significantly increased participation in some cognitively stimulating activities (eg, reading books; Figure 5). This analysis tested whether the observed data (scores on the activity protocols) showed a linear trend.

There were no significant behavior modifications with respect to other cognitively stimulating activities of the weekly activity protocols like "played a game with others," "worked at the PC," "done gardening or crafts," "played a group or individual sport," "participation in further training courses or excursions," "walked," "went by bicycle," "done memory training," "made music," and "made phone calls."

Overall, the standardized psychometric tests and questionnaires indicated no significant intervention effects (Table 5). Cognition, mood, and attitude to old age remained stable over the 6-month period, and the combination of AKTIVA-MCI and exercise (intervention group 2) was not more effective than the AKTIVA-MCI program alone (intervention group 1).

\section{Discussion}

This study aimed to investigate the feasibility of an interactive training program for patients with MCI. The results of this study support the initial hypothesis that the program is feasible and that participants are motivated and capable of participating in this comprehensive program. The evaluation

Table 4 Results of the trend analysis

\begin{tabular}{llll}
\hline Dependent variable & $\mathbf{B}_{\text {const }}$ & $\mathbf{B}_{\text {int }}$ & $\boldsymbol{P}_{\text {-value }}$ \\
\hline Watched \\
Read a book & 0.19 & -0.02 & 0.00 \\
Read a newspaper & -0.13 & 0.02 & 0.05 \\
Read a magazine & -0.06 & 0.01 & $<0.01$ \\
Played a game alone (eg, solitaire) & 0.14 & -0.02 & $<0.01$ \\
Yoga & -0.29 & 0.03 & 0.00 \\
Social interaction & -0.16 & 0.02 & $<0.01$ \\
\hline
\end{tabular}

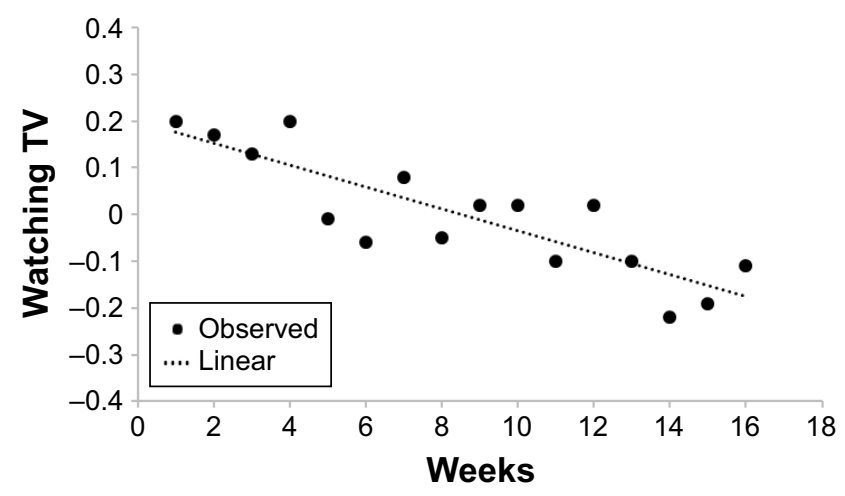

Figure 4 Trend analysis for the activity item "watched light entertainment on TV."

of the training shows that the majority of participants enjoyed the AKTIVA-MCI program. All participants were pleased with the training and would recommend it to others. The majority completed the whole program. Only six participants did not complete the study because of illness and hospitalization $(n=3)$ and time stress $(n=3)$. The cause of withdrawal due of time stress was the travel duration to the location of the AKTIVA-MCI training sessions (in some cases, $>90$ minutes). This should be considered for the planning of future training session.

The results also indicated that the training content communicated to participants was successfully implemented in their daily life routine. This is supported by significant behavioral modifications during the intervention period: between the group sessions, the participants participated in more cognitively stimulating leisure activities and reduced participation in adverse activities. Furthermore, standardized psychometric tests and questionnaires showed that measures of cognition, mood, and attitude to old age remained stable over the 6-month period.

These findings indicate that the program is suitable for patients with MCI and may contain benefits for them. This conclusion is further supported by our previous finding that

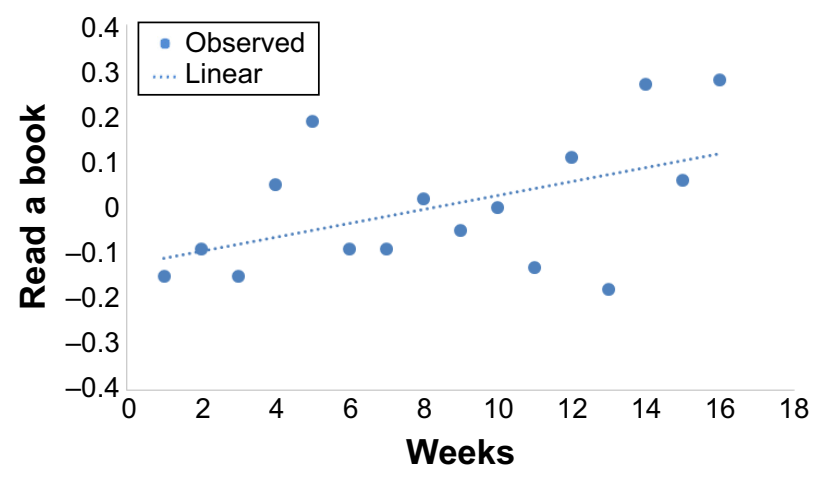

Figure $\mathbf{5}$ Trend analysis of the activity item "read a book." 
Table 5 Pre-post scores and intervention effects of the pooled sample $(\mathrm{N}=29)$

\begin{tabular}{|c|c|c|c|c|c|c|c|}
\hline $\begin{array}{l}\text { Independent } \\
\text { variable }\end{array}$ & Dependent variable & Pre & Post & df & $\boldsymbol{F}$ & $P$-value & $\eta^{2}$ \\
\hline \multirow[t]{9}{*}{ Time } & MMSE, mean (SD) & $28(2.2)$ & $28(1.5)$ & $1 / 28$ & 0.0 & 0.00 & 0.00 \\
\hline & MMSE, range & $22-30$ & $25-30$ & & & & \\
\hline & ADAS-Cog, mean (SD) & $8.1(3.7)$ & $7.8(3.5)$ & $\mathrm{I} / 28$ & 0.3 & 0.61 & 0.01 \\
\hline & ADAS-Cog, range & $2-18$ & $2-17$ & & & & \\
\hline & SDS, mean (SD) & $36.7(8.61)$ & $36.3(8.4)$ & $1 / 28$ & 0.7 & 0.42 & 0.03 \\
\hline & NSL, mean (SD) & 47.1 (II.5) & $46.2(10.9)$ & $1 / 28$ & 0.2 & 0.66 & 0.01 \\
\hline & MAC-Q, mean (SD) & $26.2(4.5)$ & $26(4.8)$ & $1 / 28$ & 0.0 & 0.93 & 0.00 \\
\hline & PGCMS, mean (SD) & II.5 (4.I) & $10.9(3.8)$ & $\mathrm{I} / 28$ & 0.5 & 0.48 & 0.02 \\
\hline & LBZ, mean (SD) & $25.7(4.2)$ & $26.5(4.1)$ & $\mathrm{I} / 28$ & 0.9 & 0.35 & 0.04 \\
\hline
\end{tabular}

Abbreviations: MMSE, Mini Mental Status Examination; ADAS-Cog, Alzheimer's Disease Assessment Scale cognitive subscale; SDS, Self-Rating Depression Scale; NSL, Nuremberg Self-Rating List; MAC-Q, Memory Complaint Questionnaire; PGCMS, Philadelphia Geriatric Center Morale Scale; LBZ, quality-of-life scale; df, degree of freedom.

a complex intervention combining AKTIVA-MCI with omega-3 fatty acid intake and aerobic exercise prevents atrophy in AD-related brain regions compared to omega-3 fatty acid intake plus a control condition of stretching and toning. ${ }^{21}$

The results of our pilot study encourage to further investigate the efficacy of the program within a larger randomized controlled trial. Based on the four factors identified by Kasper et al, AKTIVA-MCI fulfills all four requirements that may determine the efficacy of a cognitive rehabilitation program: 1) It takes special account of the individual needs of participants. ${ }^{10}$ Every session includes time to relate individual experiences and exchange information. Every exercise in the program, for example, defining individual health status, goal setting by drawing a picture, or defining motivational strategies, considers the personal needs and interests of the participants. Furthermore, every participant has the chance to take part in individual coaching sessions and to talk about their individual approaches to increasing participation in cognitively stimulating activities in their leisure time. 2) AKTIVA-MCI provides strategies to compensate for cognitive impairment. Attribution styles and memory management strategies are introduced, while role-play rounds off the theoretical content. 3) The program supports interaction with other patients. AKTIVA-MCI takes place in small groups, and the exchange with others is an important aspect of the program. By interacting with others and seeking solutions to everyday problems and difficulties, it is hoped that participants will internalize training content and make it part of their everyday lives on a sustainable basis, and 4) different therapeutic methods such as approaches to stress management, solution-oriented problem-solving strategies, and short-term therapies are included in the program.

In particular, intensive contact with others in small groups seems to positively influence mood and the subjective appraisal of memory decline. Participants in our program experienced sharing common difficulties with other people with MCI. Typical statements of participants were: "I'm not yet as senile as all that," "It greatly encouraged me to play a more active role in my future life," and "I became more aware that it is possible to do something against it." Such positive experiences may prevent negative emotions like anxiety, shame, and depressions, which are very often associated with cognitive decline, especially in the early phase of MCI. This could ultimately prevent social isolation and depression, which play an important role as additional risk factors in cognitive decline. ${ }^{30}$ And, last but not least, social contact and interaction are generally considered to prevent cognitive decline for a certain time. ${ }^{31}$

Numerous significant behavioral modifications demonstrated in the daily activity protocols during the intervention are most remarkable. Certain behaviors were reduced: After the program, participants watched light entertainment on TV, read magazines, and played games alone less often than before participation in the program. It is assumed that these leisure activities show little cognitive benefit. ${ }^{12,22}$ In contrast, participants significantly increased their participation in cognitively stimulating activities such as reading books and newspapers and interacting socially with others. Furthermore, the participants significantly increased the time period they practised yoga. The daily activity protocols may have contributed to these results. Webber et al has previously demonstrated that daily self-monitoring can be instrumental in encouraging desired behaviors. ${ }^{32}$

Our study cannot answer the questions whether the observed behavioral changes are sustainable and whether they lead to the prevention of further cognitive decline in the long run. These questions could only be answered by a randomized long-term study using an adequate control condition. 


\section{Limitations of the study}

There are several methodological limitations in the present study. First, the sample size was small $(\mathrm{N}=29)$. Therefore, the findings are only preliminary and do not allow generalization to other settings and populations. Furthermore, the majority of the participants were highly educated and those who participated were expected to benefit from the intervention and were prepared to invest the necessary time. Even if the sessions lasted for only 120 minutes, it often took equally long to reach the location of the sessions using public transport. Therefore, AKTIVA-MCI group sessions should be provided close to the homes of the participants, so that travel time is no longer an issue. Cooperation with local senior citizen associations would help in this respect. Most of the participants were already active in their free time, read a great deal, played sport, and were socially active. People who would probably benefit more from the intervention such as those who are not active at all and/or are from underprivileged social classes did not participate in the study. It was also not possible to attract people with a migration background.

A further limitation of our study is the lack of an adequate control group. Without a control intervention, whether the observed outcomes were due to the specific components of the intervention or to non-specific factors cannot be determined with certainty. Therefore, further studies should consider the inclusion of wait-list control groups. The daily activity protocols took some participants a great deal of time and were not completed consistently. For this reason, only 24 daily activity protocols were analyzed. Furthermore, all information on daily activities were self-reported and may have been biased by social desirability. A lie scale was not used to statistically control for this putative self-reporting bias. At least a comparison from pre- versus post- frequencies of cognitively stimulating leisure activities should have improved evaluation. Unfortunately, there are no data about pre-frequencies of cognitively stimulating leisure activities. This should be considered for future trainings.

\section{Conclusion}

Our results suggest that cognitive stimulation therapy is feasible for patients with MCI. The AKTIVA-MCI program gives motivational support and indicates that it is never too late to start participating in (new) activities. Analysis of the daily activity protocols showed some interesting and significant behavioral modifications. However, results should be interpreted carefully because of the small sample size. Replications with a representative sample and improved methodology (eg, control group) are clearly needed.

\section{Disclosure}

The authors report no conflicts of interest in this work.

\section{References}

1. Petersen RC. Mild cognitive impairment as a diagnostic entity. J Intern Med. 2004;256(3):183-194.

2. Eshkoor SA, Hamid TA, Mun CY, Ng CK. Mild cognitive impairment and its management in older people. Clin Interv Aging. 2015;10: 687-693.

3. Jean L, Bergeon M-E, Thivierge S, Simard M. Cognitive intervention programs for individuals with mild cognitive impairment: systematic review of the literature. Am J Geriatr Psychiatry. 2010;18(4): 281-296.

4. Martin M, Clare L, Altgassen AM, Cameron MH, Zehnder F. Cognition-based interventions for healthy older people and people with mild cognitive impairment. Cochrane Database Syst Rev. 2011; 19(1):CD006220.

5. Reijnders J, van Heugten C, van Boxtel M. Cognitive interventions in healthy older adults and people with mild cognitive impairment: a systematic review. Ageing Res Rev. 2013;12(1):263-275.

6. Belleville S, Gilbert B, Fontaine F, Gagnon L, Ménard E, Gauthier S. Improvement of episodic memory in persons with mild cognitive impairment and healthy older adults: evidence from a cognitive intervention program. Dement Geriatr Cogn Disord. 2006;22:486-499.

7. Papp KV, Walsh SJ, Snyder PJ. Immediate and delayed effects of cognitive interventions in healthy elderly: a review on current literature and future directions. Alzheimers Dement. 2009;5:50-60.

8. Owen AM, Hamphire A, Grahn JA, et al. Putting brain training to the test. Nature. 2010;465:775-778.

9. Kurz A, Pohl C, Ramsenthaler M, Sorg C. Cognitive rehabilitation in patients with mild cognitive impairment. Int J Geriatr Psychiatry. 2009;24:163-168

10. Kasper E, Thöne-Otto A, Bürger K, et al. Cognitive rehabilitation in early stage Alzheimer's disease. Der Nervenarzt. 2016;87(7):708-718.

11. Scarmeas N, Levy G, Tang M-X, Many J, Stern Y. Influence of leisure activity on the incidence of Alzheimer's disease. Neurology. 2001; 57:2236-2242.

12. Wilson RS, Bennett DA, Bienias JL, et al. Cognitive activity and incident AD in a population-based sample of older persons. Neurology. 2002; 59:1910-1914.

13. Dannhauser TM, Cleverly M, Whitfiedl TJ, Fletcher BC, Stevens T, Walker Z. A complex multimodal activity intervention to reduce the risk of dementia in mild cognitive impairment-ThinkingFit: pilot and feasibility study for a randomized controlled trial. BMC Psychiatry. 2014; $14: 129$

14. Tesky VA. Entwicklung, Durchführung und Evaluation eines Interventionsprogramms zur aktiven kognitiven Stimulation im Alter (AKTIVA) [Development, implementation and evaluation of an intervention training program for cognitive stimulation in the old age (AKTIVA)]. Berlin (Germany): Logos; 2010.

15. Tesky VA, Thiel C, Banzer W, Pantel J. Effects of a group program to increase cognitive performance through cognitively stimulating leisure activities in healthy older subjects (the AKTIVA-study). GeroPsych. 2011;24(2):83-92.

16. Tesky V, Pantel J. Geistige Fitness Erhalten - das AKTIVA-Programm. Manual für Pflegende und Gruppenleiter in der Seniorenarbeit [Prevention of cognitive activity - the AKTIVA program. A manual for senior citizen work]. Wien (Austria): Springer; 2013.

17. Morris JC, Heyman A, Mohs RC, et al. The Consortium to Establish a Registry for Alzheimer's Disease (CERAD). Part I. Clinical and neuropsychological assessment of Alzheimer's disease. Neurology. 1989;39(9): 1159-1165.

18. Petersen RC, Smith GE, Waring SC, Ivnik RJ, Tangalos EG, Kokmen E. Mild cognitive impairment: clinical characterization and outcome. Arch Neurol. 1999;56(3):303-308. 
19. Kühner C, Bürger C, Keller F, Hautzinger M. Reliability and validity of the Revised Beck Depression Inventory (BDI-II). Results from German samples. Nervenarzt. 2007;78(6):651-656.

20. Laux L, Glanzmann P, Schaffner P, Spielberger CD. Das State-TraitAngstinventar. Theoretische Grundlagen und Handanweisung [The state-trait-anxiety-inventory: theoretical bases and manual]. Weinheim (Germany): Beltz Test GmbH; 1981.

21. Köbe T, Witte V, Schnelle A, et al. Vitamin B12 concentration, memory performance and hippocampal structure in patients with mild cognitive impairment. Am J Clin Nutr. 2016;103(4):1045-1054.

22. Wilson RS, Scherr PA, Schneider JA, Tang Y, Bennett DA. The relation of cognitive activity to risk of developing Alzheimer's disease. Neurology. 2007;69:1-10.

23. Folstein MF, Folstein SE, McHugh PR. Mini-Mental State (a practical method for grading the state of patients for the clinician). J Psychiatr Res. 1975;12:189-198.

24. Rosen WG, Mohs RC, Davis KL. A new rating scale for Alzheimer's disease. Am J Psychiatry. 1984;11:1356-1364.

25. Zung WWK. A self-rating depression scale. Arch Gen Psychiatry. 1965; 12:63-70.

26. Crook TH 3rd, Feher EP, Larrabee GJ. Assessment of memory complaint in age-associated memory impairment: The MAC-Q. Int Psychogeriatr. 1992;4(2):165-176.
27. Oswald WD, Fleischmann UM. Das Nürnberger Altersinventar [The Nuremberg Age Inventory]. Erlangen-Nürnberg (Germany): Universität Erlangen-Nürnberg; 1991.

28. Lawton MP. Philadelphia Geriatric Center Morale Scale: a revision. J Gerontol. 1975;30:85-89.

29. Tesky VA, Sahlender S, Matura S, Roth I, Pantel J. AKTIVA-MCI Ein Trainingsmanual zur Steigerung kognitiv-stimulierender Freizeitaktivitäten für Menschen mit Mild Cognitive Impairment (MCI) [AKTIVAMCI a training program to increase the frequency of cognitively stimulating leisure activities in people with mild cognitive impairment]. Berlin (Germany): Logos; 2014.

30. Wilson RS, Krueger KR, Arnold SE, et al. Loneliness and risk of Alzheimer disease. Arch Gen Psychiatry. 2007;64:234-240.

31. Saczynski JS, Pfeifer LA, Masaki K, et al. The effect of social engagement on incident dementia: the Honolulu-Asia Aging Study. Am J Epidemiol. 2006;163(5):433-440.

32. Webber J, Schermann B, McCall C, Coleman M. Research on self-monitoring as a behavior management technique in special education classrooms: a descriptive review. Remedial Spec Educ. 1993;14: $38-56$.
Clinical Interventions in Aging

\section{Publish your work in this journal}

Clinical Interventions in Aging is an international, peer-reviewed journal focusing on evidence-based reports on the value or lack thereof of treatments intended to prevent or delay the onset of maladaptive correlates of aging in human beings. This journal is indexed on PubMed Central, MedLine,

\section{Dovepress}

CAS, Scopus and the Elsevier Bibliographic databases. The manuscript management system is completely online and includes a very quick and fair peer-review system, which is all easy to use. Visit http://www.dovepress. $\mathrm{com} /$ testimonials.php to read real quotes from published authors. 